\title{
COVID-RO study: the radiation oncology practice at times of COVID-19 outbreak - international survey
}

\author{
Sarit Appel, Yaacov Richard Lawrence, Zvi Symon, Orit Kaidar-Person \\ Department of Radiation Oncology, Sheba Medical Center, Tel HaShomer, Israel, affiliated with Sackler Faculty of Medicine, \\ Tel Aviv University, Israel
}

\begin{abstract}
Background: Radiation therapy (RT), an essential treatment of cancer, involves multiple hospital visits. We hypothesized that radiation departments would adjust their work patterns and RT protocols in response to the SARS-CoV-2 pandemic.

Materials and methods: An electronic survey was sent during April 2020 to an international sample of radiation oncologists. The survey explored various aspects of departmental preparedness, and changes to their institutional RT protocols.

Results: A total of 68 radiation oncologists from 13 countries answered the survey. Healthcare systems were at least moderately affected in $76 \%$. Most institutes appeared well prepared for the outbreak: regarding the availability of personal protective equipment, tests, and telemedicine/videoconference facilities. Screening for SARS-CoV-2 was applied in 59\% of responders. Modification of RT protocols were minor in $66 \%$, significant in $19 \%$ and no changes made in $15 \%$. The extent to which protocols were modified correlated with overall healthcare disruption $(p=0.028)$. Normal fractionation was recommended to continue in $83 \%$ and $85 \%$ of head \& neck, and cervical cancers vs. $64 \%$ of lung cancers ( $p=0.001)$.

In case the pandemic worsens, there was strong agreement to prioritize RT for aggressive cancers (80\%), delay RT for slowgrowing tumors (78\%) and change to evidance-based hypofractionations protocols (79.4\%). The option of delayed/omitted adjuvant RT (not site specific) was selected in $47 \%$.

Conclusion: This international survey concludes that, by making significant organizational adjustments and minor protocol modifications, RT may be safely continued during this pandemic. If the crisis worsens, there was strong agreement to continue the treatment of aggressive tumors and utilize evidence-based hypofractionated protocols.
\end{abstract}

Key words: radiation oncology; coronavirus; SARS-CoV-2; health care services

Rep Pract Oncol Radiother 2021;26(1):20-28

\section{Introduction}

The World Health Organization (WHO) has declared 2019 novel coronavirus disease (COVID-19), caused by SARS-CoV-2, a public health emergency of international concern [1]. The pandemic spread rapidly consuming health resources and causing confusion in public health services. Patients with comorbidities such as hypertension were found to be more susceptible to COVID-19 complications and death. Patients with cancer were suggested to be more susceptible to infection than individuals without cancer because of their systemic immunosuppressive state induced by the malignancy and anticancer treatments, such as chemotherapy, radiation therapy (RT) and surgery $[2,3]$. According to Zhang et al., 52\% of 107 cancer patients developed severe COVID-19 disease. This rate was found to be even higher among patients receiving active anticancer treatment than those being merely

Address for correspondence: Sarit Appel MD, Department of Radiation Oncology, Sheba Medical Center, Tel HaShomer, Israel, tel:(+97) 2544589436, fax: (+97) 2035305781; e-mail: sarit.appel@sheba.health.gov.il 
followed-up (64.9\% vs. $45.7 \%)$, with a corresponding impact on survival [3].

The pandemic has required health services to adjust rapidly, providing large-scale respiratory support units for patients with COVID-19, while simultaneously maintaining core health services. These adjustments include more intensive care units, personal protective equipment, manpower, telemedicine technologies to reduce ambulatory patient visits.

It is commonly accepted that cancer treatment should not be delayed. Delaying cancer treatment until resolution of the COVID-19 pandemic would be expected to compromise patients' prognosis. RT is an important clinical modality used to treat cancer patients, and is applied in $50-60 \%$ of cancer patients [4] with both curative and palliative intent. RT treatment includes multiple patient encounters with many different health professionals (physicians, simulator staff, therapists, nurses) - increasing the potential viral exposure of both staff and patients. Hence, special precautions are required for the RT departments $[5,6]$.

In this survey, we map the organization changes made by RT departments across Europe and North America. We hypothesized that RT departments would adopt moderate/extreme hypofractionation protocols to reduce patient treatment visits, and in case the crisis worsens, recommend treating only urgent palliative cases.

\section{Materials and methods}

The survey was designed by two senior radiation oncologists (SA, OPK) from Israel during March 2020. On 10th of April 2020 the survey was distributed via "Google Forms" that was sent via a network of RT oncologists in Europe and North America that had completed their professional training and were currently practicing in radiation oncology (not site specific). All authors used their personal international network, based on their membership/ /activities in the NRG organization [National Surgical Adjuvant Breast and Bowel Project (NSABP), the Radiation Therapy Oncology Group (RTOG), and the Gynecologic Oncology Group (GOG)], European Organization for Research and Treatment of Cancer - Young radiation oncology group (EORTC-YROG), American Society for Radiation Oncology (ASTRO) and European Society for Ra- diotherapy and Oncology (ESTRO), adding up to a total of 250 radiation oncologists.

Items in the questionnaire referred to different aspects of preparedness of the radiation oncology department, daily practice during the SARS-CoV-2 outbreak, physician's opinion on the changes that were currently taking place, and their anticipations if the crisis worsened. Voluntary and anonymous survey responses were received from $10^{\text {th }}$ April until $9^{\text {th }}$ of May 2020.

Per our institutional policy, this survey study did not require ethical approval.

Q1-Q2 questions addressed country of practice, and type of institution. Q3-Q13 - referred to the extent to which the health care was affected according to region and if the health care system was prepared for the outbreak including personal protective equipment (PPE), manpower, and technologies for telemedicine (Tab. 1).

Q13-14 referred to national guidance and institutional protocols for treatment of oncologic patients. Q15-16 referred to anticipated protocol modifications if the situation worsens? Q19-21 referred to specific recommendation for patients with curative cancers that are mainly treated with RT (Tab. 2).

Q17-18, Q22-24 referred to policies for screening tests of cancer patients and chain of delivering information and approach to patients living in "endemic" regions as well as treatment of cancer patients if they are diagnosed with SARS-CoV-2 infection. Q24 aimed to evaluate if institutions were prepared for the future, after the outbreak subsides (Tab. 3).

\section{Statistical analysis}

Descriptive statistics (rates, percentages) were calculated using Excel (Microsoft Corporation, Redmond, WA) software. Chi-square statistics was used to compare between groups. We defined "strong agreement" as concurrence of at least $80 \%$ and "agreement" as concurrence of at least $66 \%$.

\section{Results}

A total of $68(27.2 \%)$ completed the questionnaires from 13 countries. Forty-seven percent of answers were from the US, 30\% from Europe and $23 \%$ from Israel. Eighty-five percent were from academic institutions or institutions with university affiliation. 
Table 1. Healthcare system

\begin{tabular}{|c|c|c|}
\hline & \multicolumn{2}{|c|}{ Responders } \\
\hline & Number & Percent \\
\hline \multicolumn{3}{|l|}{ Q3. How much the healthcare system is affected (exhausted) in your region } \\
\hline A. Not so much & 16 & $23.5 \%$ \\
\hline B. Moderately affected & 35 & $51.5 \%$ \\
\hline C. Extremely affected & 17 & $25 \%$ \\
\hline \multicolumn{3}{|l|}{ Q4. In your opinion, how well did your institute prepare to deal with the COVID-19? } \\
\hline A. Very well & 33 & $48.5 \%$ \\
\hline B. Adequate, with some gaps & 33 & $48.5 \%$ \\
\hline C. Not at all & 2 & $3 \%$ \\
\hline \multicolumn{3}{|c|}{ Q5. Did you receive clear instructions of ways to protect yourself against the infections when treating oncologic patients? } \\
\hline A. Yes, instructions are very clear & 41 & $60.3 \%$ \\
\hline B. Instructions are vague & 24 & $35.3 \%$ \\
\hline C. No instructions were provided for protection & 3 & $4.4 \%$ \\
\hline \multicolumn{3}{|c|}{ Q6. Do you think the hospital has enough protective equipment (such as protective masks, gloves robes?) } \\
\hline A. Yes, plenty & 18 & $26.5 \%$ \\
\hline B. Yes, but we are encouraged to use it sparingly & 31 & $45.6 \%$ \\
\hline C. There is not enough protective equipment & 19 & $27.9 \%$ \\
\hline \multicolumn{3}{|l|}{ Q7. Is any of the staff sick or in isolation after being exposed to a COVID-19 carrier? } \\
\hline A. No & 32 & $47 \%$ \\
\hline B. Yes, less than $25 \%$ of the staff & 33 & $48.5 \%$ \\
\hline C. Yes, between 25 to $50 \%$ & 3 & $4.5 \%$ \\
\hline \multicolumn{3}{|l|}{ Q8. Did your department make changes to workload according to the staff? } \\
\hline A. Yes & 50 & $73.5 \%$ \\
\hline B. No, we continue to work the same, with a larger workload to those who come to work & 6 & $8.8 \%$ \\
\hline C. None of the staff are sick or absent & 12 & $17.7 \%$ \\
\hline \multicolumn{3}{|l|}{ Q9. Do you feel overwhelmed, overworked due to the COVID-19 pandemic? } \\
\hline A. No & 45 & $66.2 \%$ \\
\hline B. Yes, a bit & 19 & $28 \%$ \\
\hline C. Yes, significantly overwhelmed & 4 & $6 \%$ \\
\hline \multicolumn{3}{|l|}{ Q11.Are the health care personal working on separate shifts? } \\
\hline A. Yes, for the most part & 50 & $73.5 \%$ \\
\hline B. No, we do not have enough personal & 17 & $26 \%$ \\
\hline C. Many of our staff was differed to other departments & 1 & $1.5 \%$ \\
\hline \multicolumn{3}{|l|}{ Q12. Are you using telemedicine and videoconference etc. to reduce contacts? } \\
\hline A. Yes, for the most part & 62 & $95.4 \%$ \\
\hline B. No, we do not have the equipment & 3 & $4.6 \%$ \\
\hline
\end{tabular}

\section{Health care systems preparedness (Tab. 1)}

The health care system was affected extremely in $25 \%$ and moderately affected in $51.5 \%$. Most (97\%) indicated that their institution was prepared for the outbreak "very well" or "adequately with some gaps". Sixty percent replied that they had received clear in- structions on how to protect themselves against the infection, while $34 \%$ indicated that the instructions were vague. Personal protective equipment (PPE) was lacking for $28 \%$ of the responders, and $45 \%$ indicated that they were encouraged to use it sparingly.

In $48.5 \%$ of answers less than $25 \%$ of staff had been affected, while only $4.4 \%$ indicated that ap- 
Table 2. Guidelines and protocols

\begin{tabular}{|c|c|c|}
\hline & \multicolumn{2}{|c|}{ Responders } \\
\hline & Number & Percent \\
\hline \multicolumn{3}{|l|}{ Q13. Did you have any National guidance for treatment of oncology patients? } \\
\hline $\begin{array}{l}\text { A. Yes, our National Society published clear recommendations for treatment and safety } \\
\text { according to staffing and cancer type }\end{array}$ & 15 & $22 \%$ \\
\hline B. There are general recommendation, non are binding & 33 & $48.5 \%$ \\
\hline C. No, there are no National guidelines & 20 & $29.5 \%$ \\
\hline \multicolumn{3}{|l|}{ Q14. Have the radiation protocols and fractionation changed at your institution? } \\
\hline A. Yes we changed our protocols significantly & 13 & $19 \%$ \\
\hline B. We made minor changed to our protocols, only for low risk disease & 45 & $66 \%$ \\
\hline C. For now the radiation protocols are as usual, no change & 10 & $15 \%$ \\
\hline \multicolumn{3}{|l|}{$\begin{array}{l}\text { Q15. If the time shoudls come whetein it is clear that treatment protocols must be changed, } \\
\text { what modifications do you anticipate? (may check several answers) }\end{array}$} \\
\hline A. Adjuvant radiation should be delayed/omitted & 32 & $47 \%$ \\
\hline B. Prostate cancer and other slow-growing tumors should be delayed & 53 & $78 \%$ \\
\hline C. Change to hypo-fractionation as much as possible, if safety evidence applies & 57 & $79.4 \%$ \\
\hline D. Only urgent palliative treatment should be performed & 18 & $26.5 \%$ \\
\hline $\begin{array}{l}\text { E. Treatment of aggressive cancers should be continued (locally advanced head } \\
\text { and neck or lung) }\end{array}$ & 54 & $79.4 \%$ \\
\hline F. I will consider referring patients to regions of low population prevalence of COVID-19 & 9 & $13.2 \%$ \\
\hline G. All of the above & 9 & $13.2 \%$ \\
\hline \multicolumn{3}{|l|}{ Q16. Will you consider referring the patients to other region in your country or continent? } \\
\hline A. Yes, this may be a good option & 21 & $31 \%$ \\
\hline B. I would prefer to delay the treatment and treat myself in the next weeks & 33 & $48.5 \%$ \\
\hline C. This is absolutely not an option due to economical consideration & 14 & $20.5 \%$ \\
\hline \multicolumn{3}{|l|}{ Q19. What do you recommend for locally advanced lung cancer patients in this time? } \\
\hline A. Continue if facilities are available with regular fractionations & 43 & $64 \%$ \\
\hline B. Continue if facilities are available with hypofractionation & 22 & $33 \%$ \\
\hline $\begin{array}{l}\text { C. I believe they are at increased risk of complication, better not treat them } \\
\text { with radiation at this time }\end{array}$ & 0 & $0 \%$ \\
\hline D. Refer for treatment at regions that are less affected & 1 & $1.5 \%$ \\
\hline E. Treat only palliative cases & 1 & $1.5 \%$ \\
\hline \multicolumn{3}{|c|}{ Q20. What do you recommend for locally advanced head and neck cancer patients, at this time? } \\
\hline A. Continue if facilities are available with regular fractionations & 54 & $83 \%$ \\
\hline B. Continue if facilities are available with hypofractionation & 10 & $15.5 \%$ \\
\hline $\begin{array}{l}\text { C.I believe they are at increased risk of complication, better not treat them } \\
\text { with radiation at this time }\end{array}$ & 0 & $0 \%$ \\
\hline D. Refer for treatment at regions that are less affected & 0 & $0 \%$ \\
\hline E. Treat only urgent palliative cases & 1 & $1.5 \%$ \\
\hline \multicolumn{3}{|l|}{ Q21. What do you recommend for curative cervix cancer patients, at this time? } \\
\hline A. Continue if facilities are available with regular fractionations & 55 & $84.5 \%$ \\
\hline B. Continue if facilities are available with hypofractionation & 7 & $10.8 \%$ \\
\hline $\begin{array}{l}\text { C. I believe they are at increased risk of complication, better not treat them } \\
\text { with radiation at this time }\end{array}$ & 1 & $1.5 \%$ \\
\hline D. Refer for treatment at regions that are less affected & 1 & $1.5 \%$ \\
\hline E. Treat only urgent palliative cases & 1 & $1.5 \%$ \\
\hline
\end{tabular}


Table 3. Tests for SARS-CoV-2 infection and future directions

\begin{tabular}{|c|c|c|}
\hline & \multicolumn{2}{|c|}{ Responders } \\
\hline & Number & Percent \\
\hline \multicolumn{3}{|c|}{ Q17. Is there a policy for screening patients with cancer for SARS-CoV-2 infection (without any symptoms) } \\
\hline A. Yes, they are screened once & 12 & $17.6 \%$ \\
\hline B. Yes, they are screened several times & 16 & $23.5 \%$ \\
\hline C. Yes, they are screened, and their family members are as well & 12 & $17.6 \%$ \\
\hline D. No, they are not screened at all & 28 & $41.2 \%$ \\
\hline \multicolumn{3}{|l|}{ Q18. What do you recommend for cancer patients diagnosed with COVID-19? } \\
\hline A. Total quarantine, time undefined & 3 & $4.4 \%$ \\
\hline B. Delay all treatments for several weeks until cleared for treatment by specialist & 31 & $45.6 \%$ \\
\hline C. Continue treatment (if cancer is aggresive and patient is asymptomatic) & 34 & $50 \%$ \\
\hline \multicolumn{3}{|l|}{ Q22. What is your approach to patients who live in "endemic" regions? } \\
\hline A. Continue with regular precaution (surgical mask) no COVID-19 exam is needed & 21 & $31 \%$ \\
\hline B. Continue treatment but use extra precaution (N95 mask/eye shield /plastic covers) & 19 & $28 \%$ \\
\hline C. Have one sample that confirms that no active infection is present & 5 & $7.4 \%$ \\
\hline D. Multiple samples on different time should be made during the radiation course & 11 & $16 \%$ \\
\hline E. Not applicable & 12 & $17.6 \%$ \\
\hline \multicolumn{3}{|c|}{ Q10. At the present time are tests for SARS-CoV-2 infection available (may answer several options) } \\
\hline A. Yes, tests are performed for all patients and staff & 4 & $5.9 \%$ \\
\hline B. Yes, tests are performed upon request & 12 & $17.6 \%$ \\
\hline C. Tests are performed only if fulfilled predetermined criteria & 49 & $72 \%$ \\
\hline D. Only for symptomatic cases who were in contact with a known COVID-19 carrier & 12 & $17.6 \%$ \\
\hline E. No tests are available, only for cases admitted to intensive care unit & 1 & $1.5 \%$ \\
\hline \multicolumn{3}{|c|}{$\begin{array}{l}\text { Q23. At you institute who is responsible for informing the department of the tests results of SARS-CoV-2 } \\
\text { during a course of radiation treatment }\end{array}$} \\
\hline A. The patient himself/herself & 12 & $17.6 \%$ \\
\hline B. The health care authorities & 11 & $16.2 \%$ \\
\hline C. The hospital where I work & 34 & $50 \%$ \\
\hline D. I do not know & 11 & $16 \%$ \\
\hline \multicolumn{3}{|c|}{ Q24. At you institute how is information of the SARS-CoV-2 infection of your patients during radiation transmitted? } \\
\hline A. The information in given in fast and effective way & 44 & $64.7 \%$ \\
\hline B. Information in given after several days, but I will defenitly be informed & 12 & $17.6 \%$ \\
\hline C. Information may not be available to us & 4 & $6 \%$ \\
\hline D. I do not know & 8 & $11.8 \%$ \\
\hline \multicolumn{3}{|c|}{ Q25. Did your institute prepare a plan for the period after the COVID-19 outbreak subsides? } \\
\hline A. Yes. We have concrete planes and made lists of patients that are on "waiting lists" & 26 & $39 \%$ \\
\hline B. We discussed this but only in general ideas & 27 & $40 \%$ \\
\hline C. I do not think this was discussed & 14 & $21 \%$ \\
\hline
\end{tabular}

proximately $25-50 \%$ of staff had been affected by COVID-19.

Modification to workload according to staff was reported by $73.5 \%$ of the responders. Most responders indicated that they were working on separate shifts (capsule/bubble teams). A quarter of the respond- ers indicated that capsules teams were not feasible due to staff shortages. Most radiation oncologists did not feel significantly overwhelmed at the time of the study (94\%) and most of the responders reported the new use of telemedicine and/or videoconference for the follow up of ambulatory patients (95\%). 


\section{Radiation treatment protocols modifications (Tab. 2)}

RT national guidelines for radiation treatment at time of COVID-19 pandemic were clear only in $22 \%$, while most answered that they were general (48.5\%) or absent (29.5\%).

Sixty-six percent of the responders indicated that minor changes had been made to RT protocols at their instution, and only $19 \%$ indicated significant changes had been made to clinial protocols. The degree of changes to RT protocols correlated with the extent that the healthcare system had been affected by the pandemic $(\mathrm{p}=0.028)$ (Fig. 1$)$.

For locally advanced lung cancer, $64 \%$ recommanded to continue with regular fractionation,

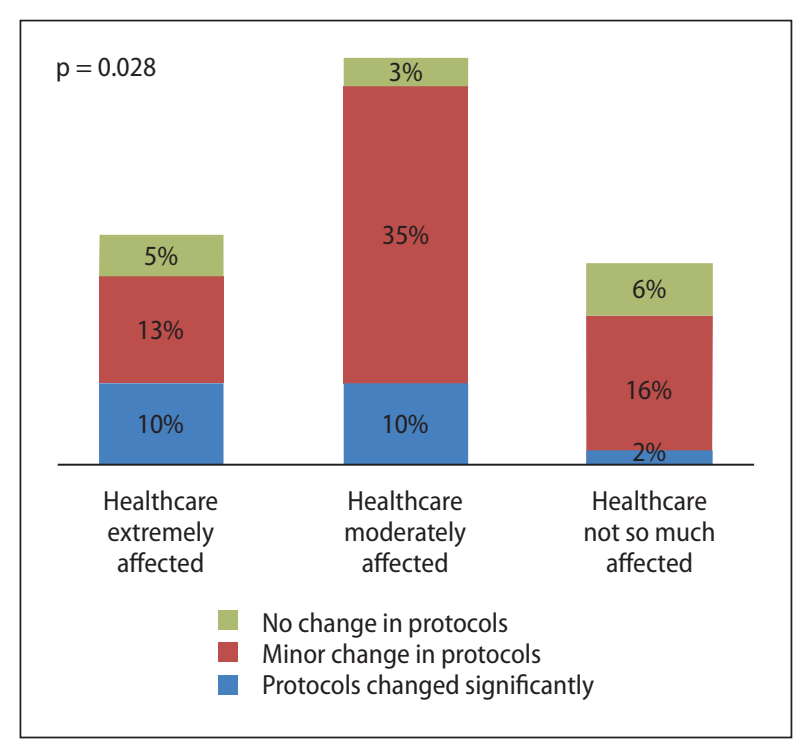

Figure 1. Protocols modification according to health care whereas 33\% opted for hypofractionation. For head and neck cancers, $83 \%$ of the responders preffered to continue with regular fractionation and only $15 \%$ opted for hypofractionation. For cervical cancers $85 \%$ of the responders preffered to continue with regular fractionation and only $11 \%$ opted for hypofractionation. Significantly more responders were reluctant to adopt hypofractionation for head and neck and cervical cancer as compared to lung cancer ( $p=0.001)$ (Fig. 2).

The responders were then asked to predict what modifications were anticipated to RT clinical protocols in case the pandemic worsens (Fig. 3). There was a strong agreement that RT of aggres-

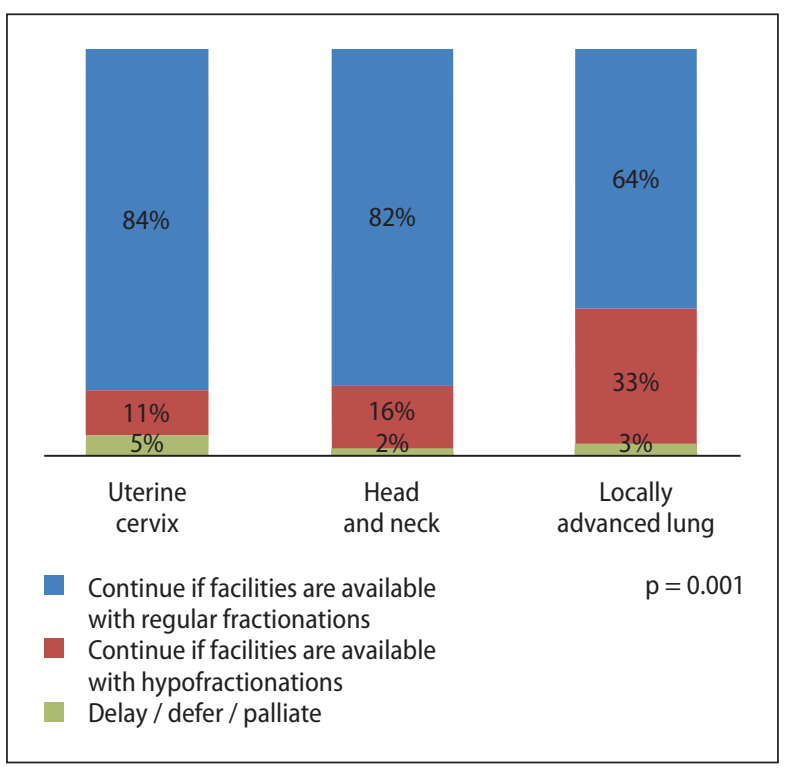

Figure 2. Current recommendation for fractionation

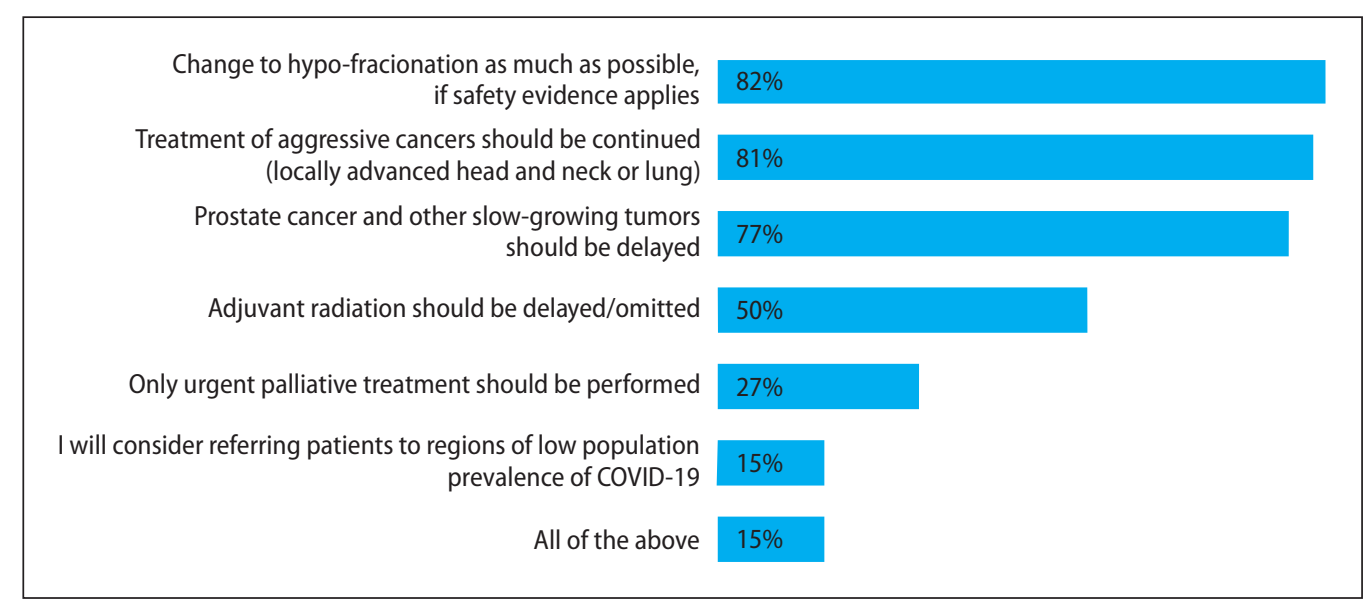

Figure 3. Anticipated protocol changes if pandemic worsens 
sive cancers should continue (80\%) and RT of low risk cancers and slow-growing tumors could be delayed (78\%). There was also a strong agreement that hypofractionation protocols should be adopted (79.4\%). The option of delayed/omitted adjuvant RT (not site specific) was selected in $47 \%$. Refering patients to departments in geographically less COVID affected regions was considered only by a third of the responders. Likewise, the option of allocating RT only for urgent palliative cases was selected only by a minority of the responders (27\%).

\section{Screening tests for SARS-CoV-2}

(Tab. 3)

A policy of screening cancer patients for SARSCoV-2 was confirmed by $59 \%$ of reponders (to different degrees), while $41 \%$ indicated that there was no such policy. Most responders indicated that screening was performed if patients fulfilled pre-determined criteria or if there were suspicious symptoms or history of contact with a COVID-19 carrier (89.6\%). Screening tests for all patients and staff was reported by only $6 \%$.

For patients who live in "endemic" regions: most (58\%) indicated that the policy was to continue RT with PPE, either regular or extra hygenic precautions, and only $23 \%$ indicated that these patients were referred for a SARS-CoV-2 screening test.

RT staff were informed of test results of SARSCoV-2 infection during the course of RT by the healthcare authority (50\%) or by the hospital (16\%), while in $17.6 \%$ by the patient himself. The patient's status of SARS-CoV-2 infection was delivered fast and effectivly to the RT staff in $82.5 \%$.

If patients tested positive for SARS-CoV-2 infection, $45.6 \%$ of the radiation oncologists recommended delaying RT and 50\% to continue RT as long as the patient was asymptomatic with regards to infection and if the cancer was considered aggresive.

Finally, we inquired if the departments were prepared for the post-pandemic phase. Only 38.7\% answered that they had made concrete plans and accumulated "waiting lists" of patients who had forfeited RT treatment during the pandemic.

\section{Discussion}

The survey was performed early after the pandemic commenced. During this time the health services were arming themselves with respirators, allocating teams to COVID-19 patients, and limiting ambulatory health services. By now, RT departments should be better adjusted to the potential threat.

Our survey shows that most RT departments had enough PPE, the instructions for personal protection were clear, staff were working in isolated team capsules/bubbles and that telemedicine and video conference facilities were available and being used. However, PPE and virology tests were used sparingly, only if indicated, showing that the health care systems were thoughtful for the resources that might become exhausted.

RT protocols changes were minor. Only $20 \%$ of the respondents had changed their protocols significantly, which could be explained by the organization alterations that had been made - allowing for the continuation of normal services. In this survey we interogated three specifically clinical scenarios for which radiation therapy is considered curative and that considered delay of treatments to be detrimental: locally advanced lung cancer, head and neck and uterine cervical cancers. Lung cancer patients may be at a hightened risk of COVID complications if infected by the virus, while head-and-neck cancer patients pose an infection risk to staff due to their hypersalivation, tracheostomies, and use of thermoplastic masks that prevent use of patient surgical masks. On the other hand, adopting hypofractionated RT while reducing the number of clinical encounters/exposures may also increase radiation toxicity potentially reducing patient tolerance. According to our survey, most responders recommended that at the time of the survey, patients with head and neck and cervix cancers should continue treatment with conventional fractionation $(83 \%$ and $84 \%$ ) (Fig. 1). This concurs with recently published expert recommendation to treat head and neck cancer patients with regular fractionation $[7,8]$.

For locally advanced lung cancer $64 \%$ of responders opted for conventional fractionation. This is in concordance with recently published ESTROASTRO consensus statement for lung cancer during the COVID-19 pandemic, which was not available during our survey. They recommended continuation with normal fractionation in concomitant radio-chemotherapy and strongly opposed deferring curative treatment of locally advanced lung cancer, as well as palliative treatments [9]. 
In our survey, the option of hypofractionation was selected in minority of cases and was more prevalent in lung cancer $v s$. head and neck and cervical cancers. This could be explained by mucosal intolerance to hypofractionation protocols or the perceived increased risk of lung cancer patients to severe COVID-19 infection compared to other disease sites, due to the inflammation caused by radiation or the pulmonary comorbidities. This is in agreement with published reports from China that stated that lung cancer patients should be the priority group for COVID-19 prevention [10].

In contrast to our results, the French national guidelines, that are non-site specific, advise the use of hypo-fractionated radiotherapy [11]. These guidelines, that are somewhat general, may be appropriate as they are leaving room for clinical judgment, which depends on the available staff and the specific prevalence of the virus that changes in time and region.

If, indeed, resources become limited or pandemic worsens, our survey indicated strong agreement to modify protocol to use evidence-based hypofractionation RT protocols, continue treatment of aggressive cancers, and delay radiation for prostate cancer and other slow growing tumors. No agreement was achieved for delaying or omitting adjuvant radiation, most probably since many adjuvant therapies are indicated for breast cancer that can be delivered in very short course of only one week according to the fast-forward randomized trial [12] and without significant immune compromise.

\section{Treatment of radiation and intercurrent SARS-CoV-2 infection}

In our survey responses were divided between continuation of curative treatments and delay of treatment until the infection has resolved. This resembles the consensus recommendations for lung and head-and-neck cancer patients to delay the beginning of treatment of COVID-19 positive patients. Yet, in these expert recommendations, if treatment has already started and symptoms of SARS-CoV-2 infection are mild, half recommended to interrupt treatment for lung cancer. However, for head and neck, most experts agreed to continue treatment $[8,9]$.

The option of referring to a different region was not chosen by most responders. If the crisis worsens, this option should be re-considered, to allow treatment in due time and not compromise the prognosis caused by delaying curative treatment.

\section{Screening for SARS-CoV-2}

In our survey most respondents answered that cancer patients were screened, which is in line with the French recommendations [11], but the consensus about frequency of screening and its extent to family members and staff was not reached, leaving this to clinical decision in every department and region. When tests are performed for SARS-CoV-2, it is important that results are delivered fast and efficiently to the medical staff. We saw that in most cases the healthcare services are responsible for informing the radiation departments of the results. Yet, in some cases it is the patient himself that delivers a test result, which is potentially less reliable.

An important limitation of this survey is that only a quarter of answers came from regions where the healthcare system was extremely affected. This may be due to unavailability of these physicians. Nonetheless, we found a strong correlation between the adaptation of modified protocols and the magnitude of healthcare exhaustion. Most answers came from academic centers, leaving private practices underrepresented.

In conclusion, radiation oncology departments, represented in this international comprehensive survey, have prepared efficiently for the COVID-19 outbreak, allowing the RO services to continue. Nonetheless, gaps in national guidance were identified, as well as a lack of preparedness for the immediate post-pandemic period. At the time the survey was performed, there was strong agreement regarding the importance of continuing radiation treatment for aggressive cancers with regular fractionation, but if the crisis worsens, to implement hypo-fractionated protocols and delay the treatment of slow growing cancers. We need to share knowledge and experience in order to ensure the continuation of cancer care in this changing reality.

\section{Conflicts of interest}

The authors S.A, Y.R.L., Z.S., and O.K.P. certify that they have $\mathrm{NO}$ affiliations with or involvement in any organization or entity with any financial interest (such as honoraria; educational grants; participation in speakers' bureaus; membership, employment, consultancies, stock ownership, or other equity interest; and expert testimony or 
patent-licensing arrangements), or non-financial interest (such as personal or professional relationships, affiliations, knowledge or beliefs) in the subject matter or materials discussed in this manuscript.

\section{Financial disclosure}

Y.R.L. has received research support from Checkmate pharmaceuticals and Bristol-Myers Squibb. S.A. received revenue for lectures from Astra Zeneca.

\section{Funding}

This research did not receive any specific grant from funding agencies in the public, commercial, or not-for-profit sectors.

\section{References}

1. Coronavirus disease (COVID-19) pandemic. https://www. who.int/emergencies/diseases/novel-coronavirus-2019.

2. Liang W, Guan W, Chen R, et al. Cancer patients in SARS-CoV-2 infection: a nationwide analysis in China. Lancet Oncol. 2020; 21(3): 335-337, doi: 10.1016/S14702045(20)30096-6, indexed in Pubmed: 32066541.

3. Zhang $H$, Wang $L$, Chen $Y$, et al. Outcomes of novel coronavirus disease 2019 (COVID-19) infection in 107 patients with cancer from Wuhan, China. Cancer. 2020; 126(17): 4023-4031, doi: 10.1002/cncr.33042, indexed in Pubmed: 32573776.

4. Baskar R, Lee KA, Yeo R, et al. Cancer and radiation therapy: current advances and future directions. Int J Med Sci. 2012; 9(3): 193-199, doi: 10.7150/ijms.3635, indexed in Pubmed: 22408567.

5. Appel S, Kaidar-Person O, Lawrence YR, et al. The Coronavirus Pandemic in Israel: Implications for Radiation Oncol- ogy Departments. Isr Med Assoc J. 2020; 22(4): 211-213, indexed in Pubmed: 32286020.

6. Combs SE, Belka C, Niyazi M, et al. First statement on preparation for the COVID-19 pandemic in large German Speaking University-based radiation oncology departments. Radiat Oncol. 2020; 15(1): 74, doi: 10.1186/s13014020-01527-1, indexed in Pubmed: 32264908.

7. Thomson DJ, Palma D, Guckenberger M, et al. Practice recommendations for risk-adapted head and neck cancer radiotherapy during the COVID-19 pandemic: An ASTROESTRO consensus statement. Radiother Oncol. 2020; 151: 314-321, doi: 10.1016/j.radonc.2020.04.019, indexed in Pubmed: 32730830.

8. Thomson DJ, Palma D, Guckenberger M, et al. Practice recommendations for risk-adapted head and neck cancer radiotherapy during the COVID-19 pandemic: An ASTROESTRO consensus statement. Radiother Oncol. 2020; 151: 314-321, doi: 10.1016/j.radonc.2020.04.019, indexed in Pubmed: 32730830.

9. Guckenberger M, Belka C, Bezjak A, et al. Practice recommendations for lung cancer radiotherapy during the COVID-19 pandemic: An ESTRO-ASTRO consensus statement. Radiother Oncol. 2020; 146(4): 223-229, doi: 10.1016/j. radonc.2020.04.001, indexed in Pubmed: 32342863.

10. Xu Y, Liu H, Hu Ke, et al. [Clinical Management of Lung Cancer Patients during the Outbreak of 2019 Novel Coronavirus Disease (COVID-19)]. Zhongguo Fei Ai Za Zhi. 2020; 23(3): 136-141, doi: 10.3779/j.issn.1009-3419.2020.03.02, indexed in Pubmed: 32077441.

11. You B, Ravaud A, Canivet A, et al. The official French guidelines to protect patients with cancer against SARSCoV-2 infection. Lancet Oncol. 2020; 21(5): 619-621, doi: 10.1016/S1470-2045(20)30204-7, indexed in Pubmed: 32220659.

12. Murray Br, Haviland JS, Wheatley DA, et al. Hypofractionated breast radiotherapy for 1 week versus 3 weeks (FAST-Forward): 5-year efficacy and late normal tissue effects results from a multicentre, non-inferiority, randomised, phase 3 trial. Lancet. 2020; 395(10237): 16131626, doi: 10.1016/S0140-6736(20)30932-6, indexed in Pubmed: 32580883. 